BMC

Systems Biology

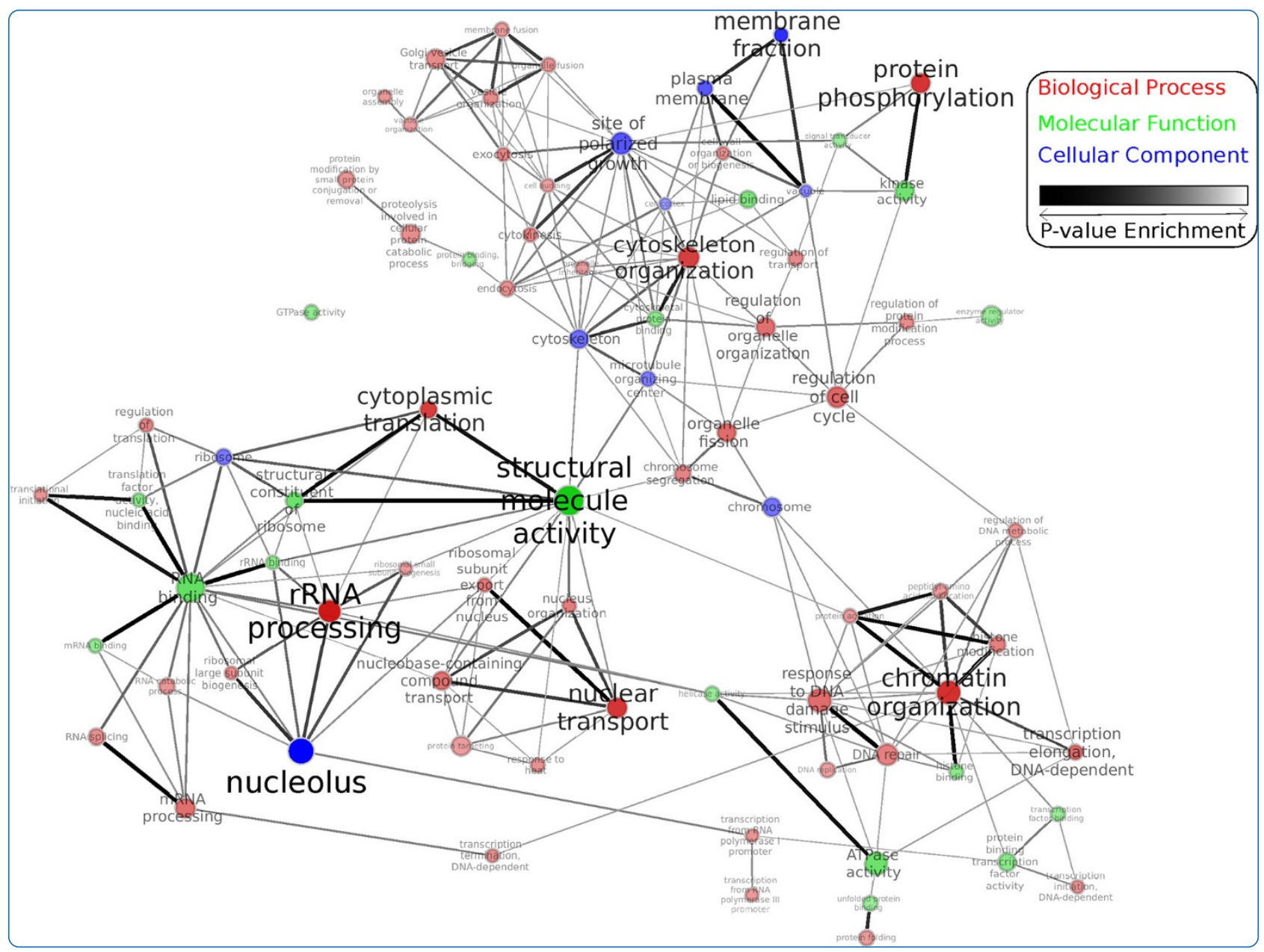

\title{
Inferring the effective TOR-dependent network: a computational study in yeast
}

Mohammadi et al. 


\title{
Inferring the effective TOR-dependent network: a computational study in yeast
}

\author{
Shahin Mohammadi ${ }^{*}$, Shankar Subramaniam² and Ananth Grama
}

\begin{abstract}
Background: Calorie restriction (CR) is one of the most conserved non-genetic interventions that extends healthspan in evolutionarily distant species, ranging from yeast to mammals. The target of rapamycin (TOR) has been shown to play a key role in mediating healthspan extension in response to CR by integrating different signals that monitor nutrient-availability and orchestrating various components of cellular machinery in response. Both genetic and pharmacological interventions that inhibit the TOR pathway exhibit a similar phenotype, which is not further amplified by CR.

Results: In this paper, we present the first comprehensive, computationally derived map of TOR downstream effectors, with the objective of discovering key lifespan mediators, their crosstalk, and high-level organization. We adopt a systematic approach for tracing information flow from the TOR complex and use it to identify relevant signaling elements. By constructing a high-level functional map of TOR downstream effectors, we show that our approach is not only capable of recapturing previously known pathways, but also suggests potential targets for future studies.

Information flow scores provide an aggregate ranking of relevance of proteins with respect to the TOR signaling pathway. These rankings must be normalized for degree bias, appropriately interpreted, and mapped to associated roles in pathways. We propose a novel statistical framework for integrating information flow scores, the set of differentially expressed genes in response to rapamycin treatment, and the transcriptional regulatory network. We use this framework to identify the most relevant transcription factors in mediating the observed transcriptional response, and to construct the effective response network of the TOR pathway. This network is hypothesized to mediate life-span extension in response to TOR inhibition.

Conclusions: Our approach, unlike experimental methods, is not limited to specific aspects of cellular response. Rather, it predicts transcriptional changes and post-translational modifications in response to TOR inhibition. The constructed effective response network greatly enhances understanding of the mechanisms underlying the aging process and helps in identifying new targets for further investigation of anti-aging regimes. It also allows us to identify potential network biomarkers for diagnosis and prognosis of age-related pathologies.
\end{abstract}

Keywords: Target of rapamycin (TOR), Yeast aging, Interactome, Information flow analysis, Effective response network

\section{Background}

Cellular aging is a multi-factorial complex phenotype, characterized by the accumulation of damaged cellular components over the organism's life-span [1]. The progression of aging depends on both the increasing rate of damage to DNA, RNA, proteins, and cellular organelles, as well as the gradual decline of cellular defense mechanisms against stress. This can ultimately lead to a dysfunctional cell with a higher risk factor for disease.

\footnotetext{
*Correspondence: mohammadi@purdue.edu

1 Department of Computer Science, Purdue University, West Lafayette, Indiana, USA
}

Full list of author information is available at the end of the article
Limiting caloric intake without causing malnutrition, also known as calorie restriction (CR), is one of the most conserved non-genetic interventions, which extends life-span in evolutionarily distant species ranging from yeast to mammals [1-3]. Inhibition of the nutrient-sensing pathways, using either genetic or pharmacological intervention, also results in a similar phenotype [1,2]. More importantly, increased lifespan is accompanied by an increased healthspan, which delays both the progression and the increasing risk-factor for a wide range of agerelated diseases, including cancers [4-7], cardiovascular disease [8-11], and multiple neurodegenerative disorders [12-17]. The extent to which these pathologies share 
their underlying biology is a topic of active investigation. Emerging evidence, however, supports the hypothesis that large classes of age-related diseases are driven by similar underlying mechanisms [18]. Understanding and controlling these mechanisms, therefore, constitute critical aspects of preventing or delaying the onset of age-related pathologies. Motivated by these observations, considerable effort has been invested in understanding the downstream effectors of the nutrient-sensing pathways that orchestrate CR-mediated life-span extension.

The budding yeast, Saccharomyces cerevisiae, has been used extensively as a model organism in aging research, due to its rapid growth and ease of manipulation $[3,19]$. Having two different aging paradigms - replicative lifespan (RLS), defined as "the number of buds a mother cell can produce before senescence occurs", and chronological life-span (CLS), defined as "the duration of viability after entering the stationary-phase", yeast provides a unique opportunity for modeling both proliferating and post-mitotic cells. Understanding the underlying mechanisms driving RLS and CLS can ultimately be used to shed light on the progression of cancers and neurodegenerative diseases, respectively.

Yeast cells are typically cultured in growth media containing $2 \%$ glucose. Reducing glucose concentration to $0.5 \%$ or less is one of the best characterized CR regimens in yeast, which increases both CLS and RLS [20-22]. The target of rapamycin (TOR) has been shown to play a key role in mediating the observed life-span extension in response to CR [23]. TOR is a serine/threonine protein kinase, which belongs to the conserved family of PI3K-related kinases (PIKKs). It was first identified using genetic studies in yeast while searching for mutants that confer rapamycin-resistance [24]. It was later discovered that TOR protein kinases, encoded by TOR1 and TOR2 genes in yeast, form two structurally and functionally distinct multiprotein complexes [25-28]. TOR Complex 1 (TORC1) is rapamycin-sensitive and consists of both TOR proteins, TOR1 and TOR2, together with KOG1, LST8, and TCO89. On the other hand, TOR Complex 2 (TORC2) does not contain TOR1, is not inhibited by rapamycin, and contains AVO1, AVO2, AVO3, LST8, BIT2, and BIT61. These two complexes correspond to two separate branches of the TOR signaling network, controlling the spatial and temporal aspects of cell growth, respectively, which are conserved from yeast to humans [28]. Interestingly, TORC1 also has a critical role in aging and age-related pathologies [29,30]. Many of the known oncoproteins act as upstream activators of TORC1, while several tumor suppressor proteins inhibit its activity [31,32]. From a systems point of view, TORC1 acts as a hub that integrates various nutrient and stress-related signals and regulates a variety of cellular responses [33-35]. Inhibiting TOR signaling using rapamycin provides a unique opportunity to identify its downstream effectors. However, these targets may be regulated in different ways, including, but not limited to, transcription regulation, translational control, and post-translational modifications. Capturing various changes that happen during rapamycin treatment, in order to create a comprehensive systems view of the cellular response, is a complex task.

In this paper, we propose a complementary, computational approach to reconstruct a comprehensive map of TOR downstream effectors. We develop a systematic approach to couple random walk techniques with rigorous statistical models, integrate different datasets, and identify key targets in calorie restriction that are mediated by TOR pathway. Using GO enrichment analysis of high scoring nodes, we show that information flow analysis not only identifies previously known targets of TORC1, but also predicts new functional roles for further studies. We cross-validate our results with transcriptome profile of yeast in response to rapamycin treatment and show that our method can accurately predict transcriptional changes in response to TORC1 inhibition. Information flow scores provide an aggregate ranking of proteins, with respect to their relevance to the TOR signaling pathway, and are highly susceptible to degree bias. To remedy this and to elucidate the roles of underlying signaling elements, we propose a novel statistical framework for integrating information flow scores, data on regulatory relationships, and the expression profile in response to rapamycin treatment.

Using our framework, we identify the most relevant transcription factors and construct the effective response network of TOR, which is responsible for the observed transcriptional changes due to TOR inhibition. Our approach, unlike experimental methods, is not limited to specific aspects of cellular response. Rather, it predicts transcriptional changes, as well as post-translational modifications in response to TOR signaling. The resulting interaction map greatly enhances our understanding of the mechanisms underlying the aging process and helps identify novel targets for further investigation of antiaging regimes. It also reveals potential network biomarkers for diagnoses and prognoses of age-related pathologies and identifies mechanisms for control of cellular aging processes through multi-targeted and combinatorial therapies [36,37].

\section{Results and discussion Computing information flow scores from TORC1}

Given the yeast interactome, constructed using the procedure detailed in Methods Section and illustrated in 
Figure 1, we compute information flow scores using random walks initiated at selected nodes in the interactome. These nodes comprise members of the TORC1 complex, each of which propagates a unit flow (normalized to 0.2 for each of the five member proteins). We use a discrete random-walk process in which, at each step, every protein aggregates incoming signals and distributes them equally among outgoing neighbors. The final information flow scores are computed as the steady-state distribution of the random-walk process. One of the key parameters in the random-walk process, which controls the depth of propagation, is called the restart-probability. This is the probability that a random walker continues the walk (as opposed to teleporting to a node chosen from among a set of preferred nodes). In order to give all nodes in the interactome a chance of being visited, we use the relationship between restart probability and the mean depth of random-walks by setting parameter $\alpha$ to be equal to $\frac{d}{1+d}$, where $d$ is the diameter of the interactome. For the yeast interactome, we determine the diameter to be equal to 6 and set $\alpha=\frac{6}{7} \sim 0.85$, correspondingly (please see the Methods section for details of information flow computations). Figure 2 illustrates the distribution of computed information flow scores, starting from TORC1, as a function of node distance from TORC1. It is evident from the figure that computed scores are functions of both distance from source nodes, as well as multiplicity of paths between source and sink nodes. This can be verified from the overlapping tails of distributions for nodes at different distances, as well as the varied distribution of scores among nodes at the same distance from TORC1. The final list of computed information flow scores is available for download as Additional file 1.

Node rankings from the random walk process are susceptible to degree-bias, favoring high-degree nodes. To remedy this bias and to gain a detailed mechanistic understanding of the roles of various proteins (and associated signaling elements), random walk methods need to be coupled with appropriate statistical tests. A key contribution of our work is the development of such a test, which yields a fine-grained understanding of key pathways involved in orchestrating cellular response to TOR inhibition. To the best of our knowledge, this work represents the first application of information flow methods for reconstructing the effective response network of TORC1.

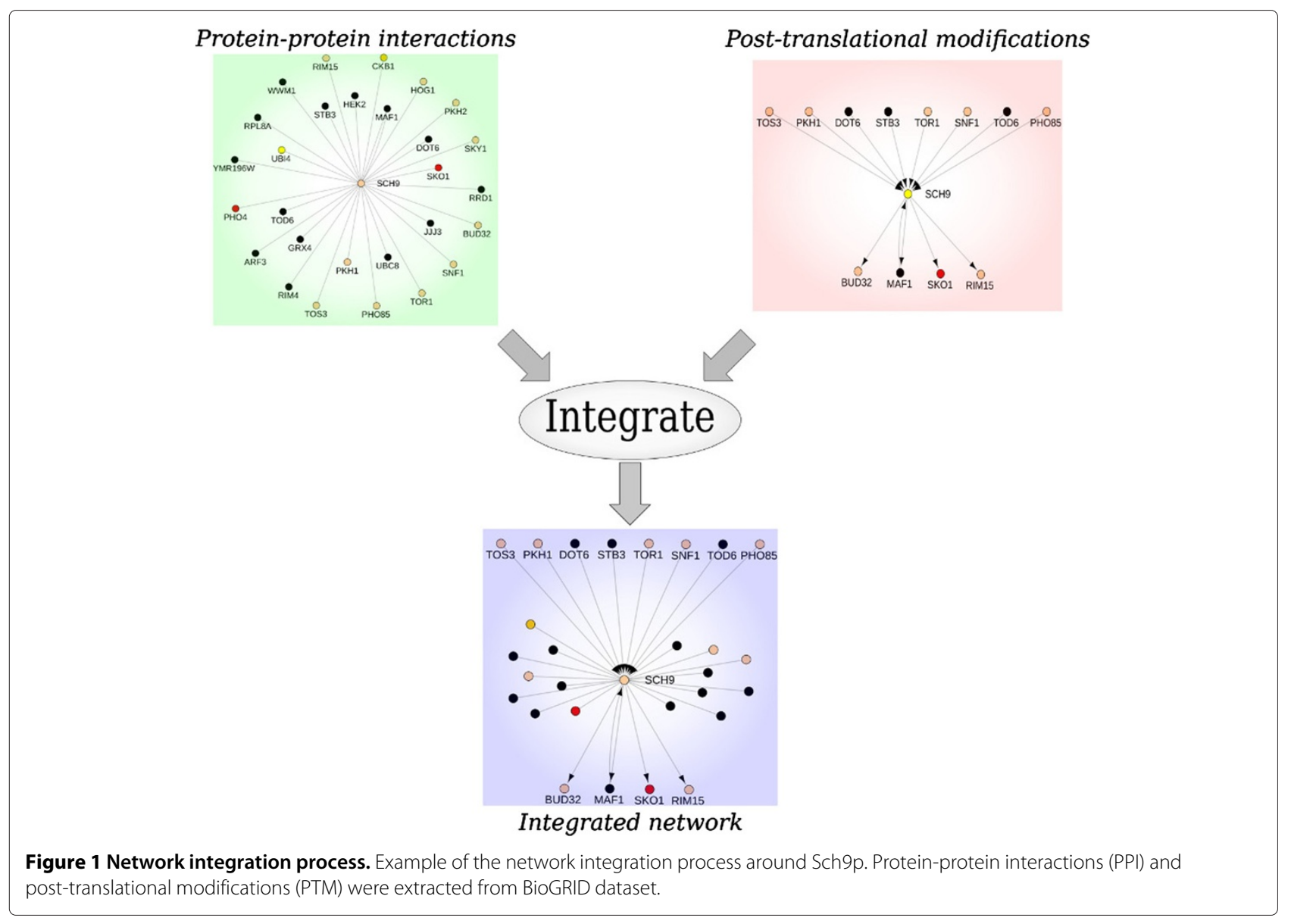




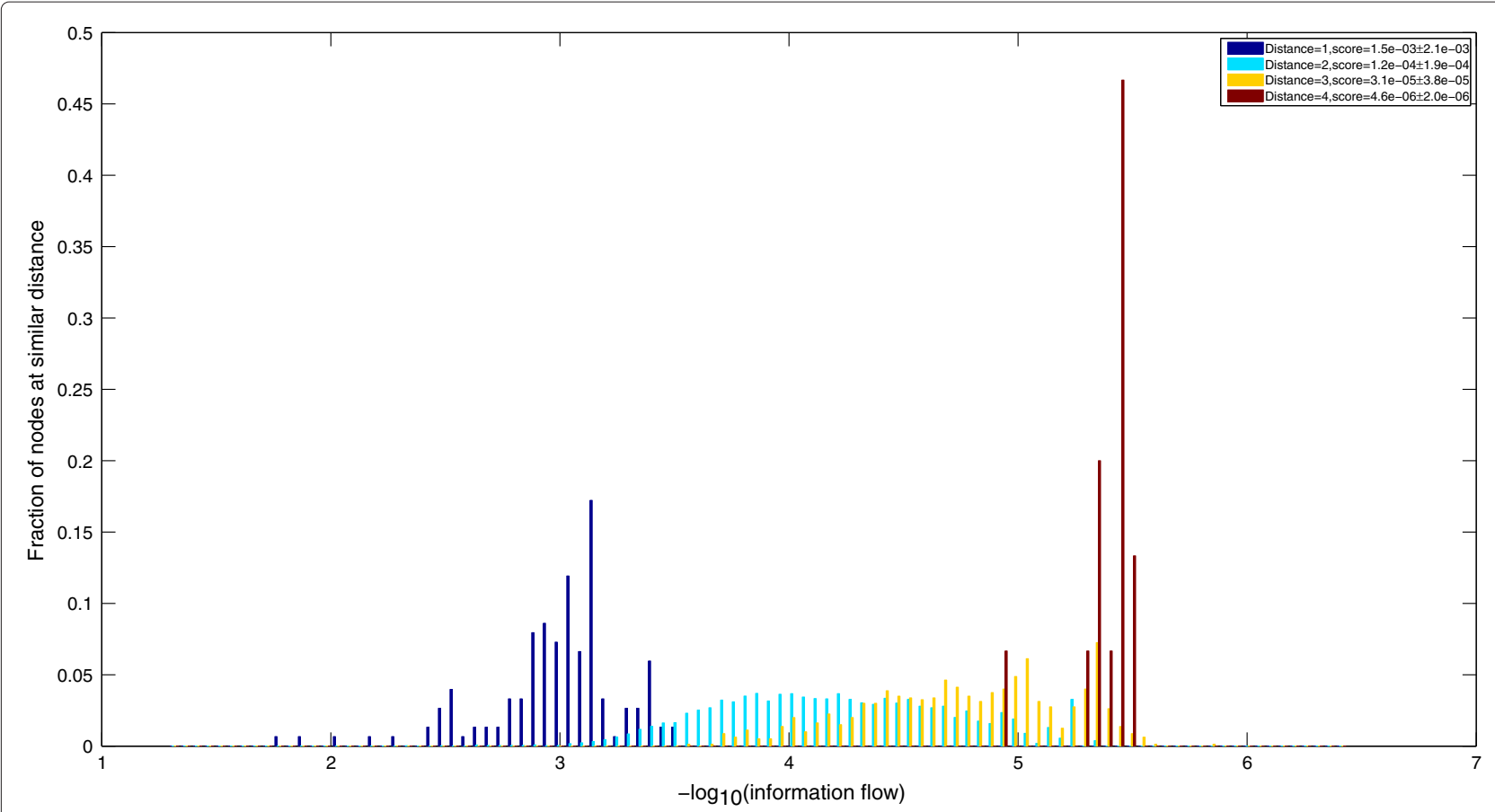

Figure 2 Distribution of random-walk scores. Information flow versus node distance from TORC1, showing that random-walk scores are a function of distance, as well as multiplicity of paths.

\section{Constructing a high-level functional map of TOR downstream effectors}

TORC1 is not only regulated by the quality and the quantity of both carbon and nitrogen sources [34,38-40], but also by noxious stressors, such as caffeine $[41,42]$. In response, TORC1 coordinately orchestrates various aspects of cellular machinery to mediate cell growth $[32,40]$. This includes autophagy [43], stress response $[42,44]$, and protein synthesis (by regulating ribosome biogenesis [45], translation initiation [46], and nutrient uptake $[47,48])$.

In order to systematically identify the functional aspects relevant to TOR signaling, we first rank the proteins in the yeast interactome based on their information flow scores from the TORC1 complex. Given this ranked list, we aim to identify functional terms that are highly overrepresented among top-ranked proteins. Gene Ontology (GO) [49] enrichment analysis has been used extensively for this purpose. We employ GOrilla [50] to find the optimal cut for each GO term, together with its exact minimum hypergeometric ( $\mathrm{mHG}$ ) p-value. Next, we enforce a threshold of $p$-value $\leq 10^{-3}$ to identify the significant terms. The complete list of enriched terms for each branch of GO is available for download as Additional files 2 .

GO provides a hierarchical vocabulary to annotate biological processes (BP), molecular functions (MF), and cellular components (CC). This hierarchical structure, represented using a directed acyclic graph (DAG), introduces an inherent dependency among the significant terms identified by GO enrichment analysis. Furthermore, seemingly independent terms under different branches of GO may be used to annotate the same set of genes. To provide a compact, non-redundant representation of the significant terms in our experiment, we follow a two-step process. First, we extract the subset of enriched terms that are marked by the Saccharomyces Genome Database (SGD) [51] as GO slim. Yeast GO slim is a compact subset of the entire GO, selected by SGD curators, which is necessary and sufficient to describe different aspects of yeast cellular biology. Next, we use EnrichmentMap (EM) [52], a recent Cytoscape [53] plugin, to construct the network (map) of the enriched terms. In this network, unlike the original interactome, each node represents a significant GO slim term and each weighted edge indicates the extent of overlap between genesets of their corresponding terms. We use a custom visualization style to illustrate various network properties. GO terms under BP, MF, and $\mathrm{CC}$ branches are color-coded red, green, and blue, respectively. The p-value of each term determines the opacity of both the node and its label; the bolder a term appears, the more significant its enrichment score. Finally, the total number of enriched genes for each GO term is shown using the size of the corresponding node. The final map, which is shown in Figure 3, is available for download as Additional file 3. This map provides unique opportunities 
for studying TOR-dependent terms visually, since terms (nodes) representing relevant sets of genes tend to cluster together in this network.

First, we note that most of the previously known targets of TORC1 are also identified by our information flow method, as represented in the enrichment map. For example, all terms related to ribosome biogenesis, including relevant cellular components (such as ribosome and nucleolus), molecular functions (such as rRNA binding and structural constitute of ribosome), and biological processes (such as rRNA processing and ribosomal subunit export from nucleus), are clustered in the bottom-left corner of the map. These terms, interestingly, are also clustered with other terms related to protein synthesis, such as regulation of translation, translational initiation, and cytoplasmic translation. Furthermore, many of the terms related to stress-response, such as response to DNA damage stimulus and DNA repair, are clustered in the bottom-left corner of the map. Finally, many of the terms related to TOR signaling, nutrient uptake, and cytoskeleton organization are grouped on the top section of the map.

Additionally, we observe that there are terms in this map that have not been adequately investigated in previous efforts. For example, even though translational control is a well-known function of TORC1, transcriptional control is less-studied. Several terms related to transcription initiation and elongation are enriched in our analysis, as shown on the bottom-right of the map. In order to gain a mechanistic understanding of these terms, we project the geneset of each term (node) back to the original network and construct the corresponding induced subgraph in the yeast interactome. As a case study, we extract the set of enriched genes represented by the transcription initiation GO term and construct its induced subgraph, which is shown in Figure 4. Here, nodes, representing proteins, are

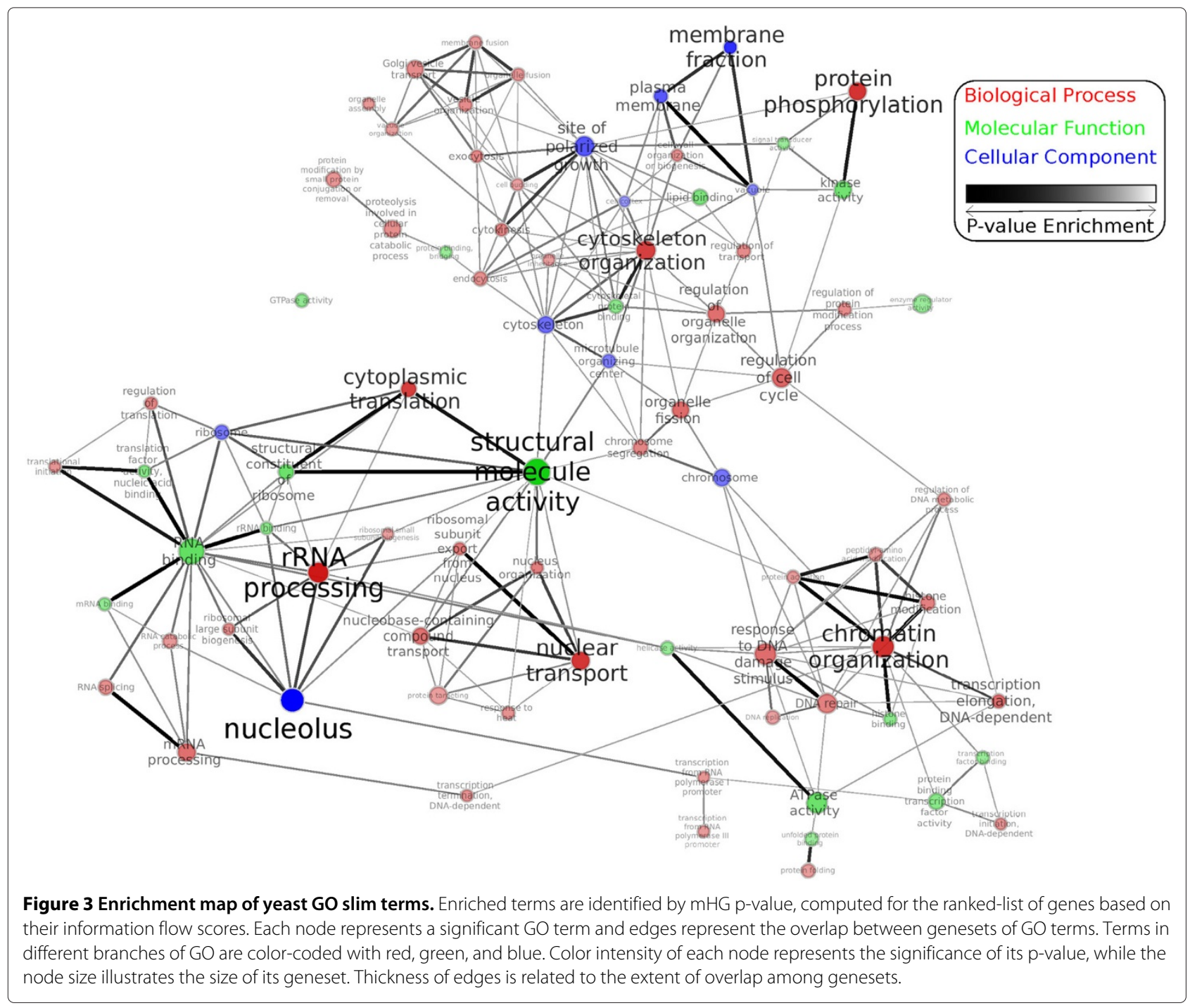


grouped and annotated based on their functional role in forming the transcription pre-initiation complex (PIC), as well as the RNA polymerase (RNAP). The basal level of transcription in Eukaryotic cells by RNAP needs a family of general transcription factors (GTF), prior to the formation of PIC. The TATA-binding protein (TBP), encoded by the Spt15 gene in yeast, is a universal GTF that is involved in transcription by all three types of nuclear RNAP. As a component of TFIIIB complex, it forms the PIC complex and recruits RNAPIII to the transcriptional start site(TSS) of tRNAs, 5S rRNA, and most snRNAs. As a part of TFIID, it forms a complex together with TBP-associated factors (TAF) and binds to the core promoter region of the protein-coding genes, as well as some snRNAs. The correct assembly of PIC, required for directing RNAPII to the TSS, needs additional GTFs, namely TFIIA, -B, -D, -E, -F, and TFIIH, as well as the Mediator (MED) complex. These components are assembled in an orderly fashion to form the PIC and mediate the transcription initiation by RNAPII (please see Hampsey [54] and Maston et al.

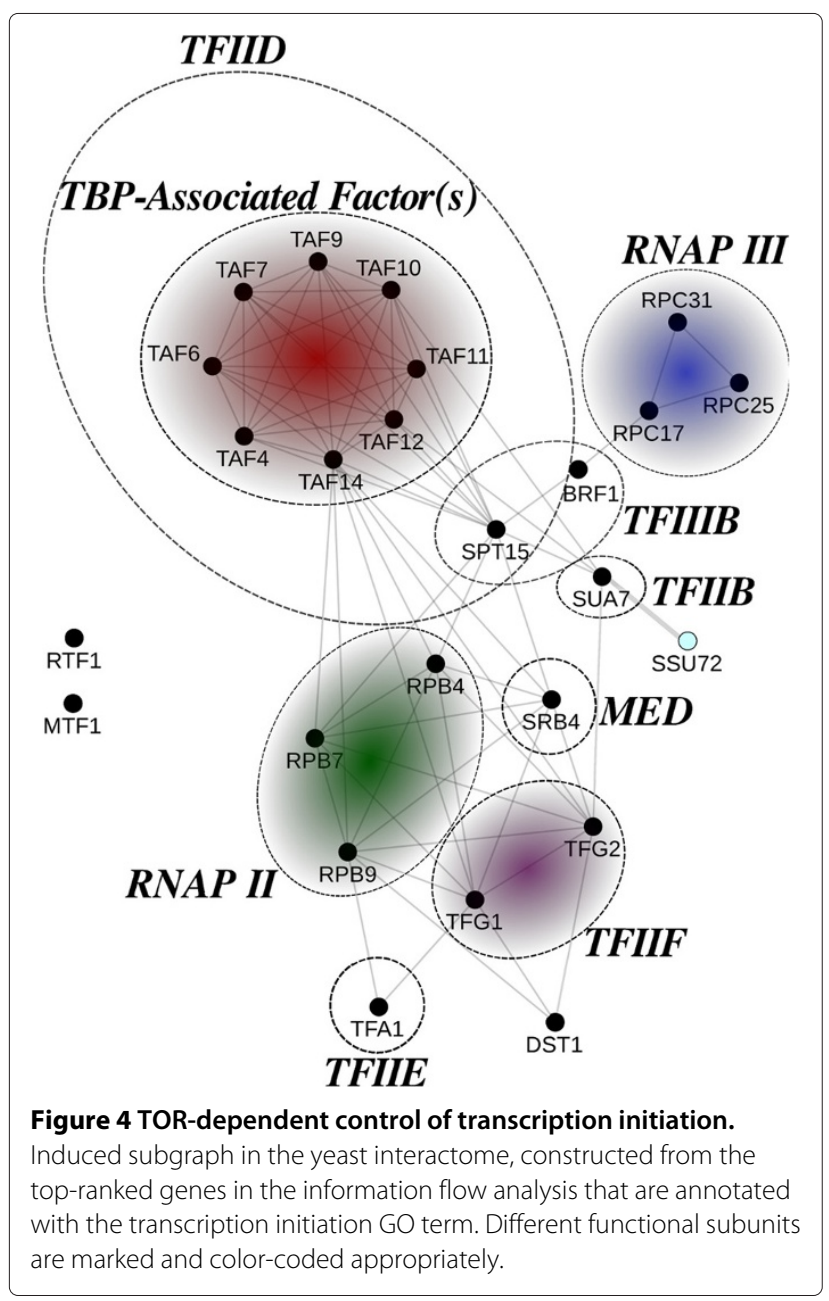

[55] for a review). These complex interactions are faithfully reconstructed in Figure 4, which provides a more refined understanding of transcription initiation, under TOR control, in the yeast cells.

\section{Comparison of predicted targets to the set of differentially} expressed genes in response to Rapamycin treatment

Rapamycin, a lipophilic macrolide originally purified as an antifungal agent and then re-discovered as an immunosuppressive drug, forms a toxic complex with its intracellular receptor FKBP12, encoded by the Fpr1 gene in yeast, and directly binds to TOR in order to perform its inhibitory action [32]. We hypothesize that if the information flow-based method agrees with the TORC1 signaling network, it should be able to predict transcriptional changes due to rapamycin treatment, which inhibits TORC1 in vivo. To validate this hypothesis, we used a recent mRNA expression profile of yeast in response to rapamycin treatment [56]. We extracted the set of differentially expressed genes, at a minimum threshold of 2 -fold change, and constructed a vector of true positives from this set by filtering out genes that do not have a corresponding vertex in the yeast interactome. The final dataset includes 342 repressed and 237 induced genes in our experiment.

Using this set of true-positives, we computed the enrichment plot of information flow scores by ranking all proteins and computing the hypergeometric score as a function of the protein rank, which is illustrated in Figure 5. The peak of the plot, corresponding to the minimum hypergeometric (mHG) score, occurs at the index $l=906$ from the top, which covers approximately the top $15 \%$ of scores. There are 181 positive genes in this partition, from a total of 579 positives, yielding a mHG score of $1.11 * 10^{-22}$. We computed the exact p-value corresponding to this $\mathrm{mHG}$ score, using the dynamicprogramming method of Eden et al. [57], resulting in the significant enrichment p-value of $3.25 * 10^{-19}$. This in turn supports our hypothesis that the random-walk neighborhood of TORC1 is highly enriched with the set of genes that are differentially expressed in response to rapamycin treatment.

\section{Post-translational modifications among top-ranked proteins: a case study on Gap1 regulation}

An interesting observation from Figure 5 is that the highest-ranked genes (approximately the top 150 genes), marked with a red box, are not enriched in terms of rapamycin-induced genes. This can be explained by the fact that regulatory elements in the TOR signaling pathway, including TFs, do not typically change their expression level in response to TOR signaling. Instead, they are targeted for post-translational modifications (typically, phosphorylation). We consequently 


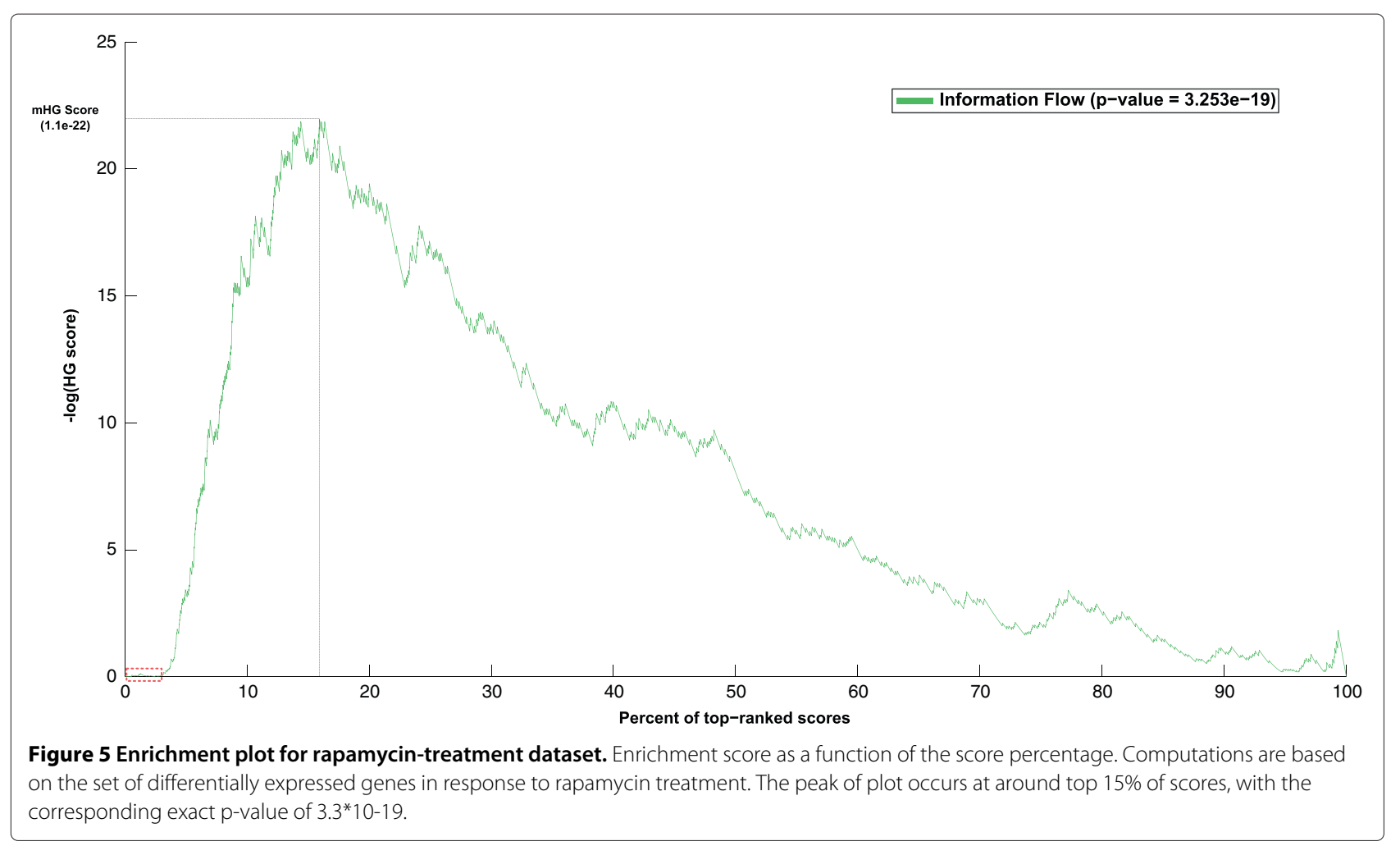

hypothesize that the top genes should also be enriched in terms of phosphorylation events. To further investigate this hypothesis, we focus on a case study of Gap1 regulation, a general amino acid permease regulated by NCR. We choose Gap1 since its regulatory pathway, originating from TORC1, is well-studied in literature. Moreover, data from phosphoproteomic experiments, which measures phosphorylation events among elements of this pathway, is readily available. Specifically, Gap1 is positively regulated via Gln3 and Gat1, while it is repressed by Gzf3 and Dal80 [34,40]. Interestingly, all four of these regulators are among top-ranked transcription factors, yet none of them are differentially expressed in response to rapamycin treatment. Using a recent phosphoproteome of yeast in response to rapamycin treatment [58], we validated that both of the transcriptional activators of Gap1, namely Gln3 and Gat1, are highly phosphorylated in response to rapamycin treatment. Moreover, Tap42-Sit4, which is the upstream regulator of $\mathrm{Gcn} 4$, is indirectly regulated by TORC1.

Figure 6 illustrates this signaling pathway, with each element annotated using its information flow rank. All signaling elements upstream of Gap1 are present among topranked scores, yet none of them change their expression levels in response to rapamycin treatment. This partially supports our hypothesis that the top-ranked genes in the random-walk are primarily targets of post-translational modifications. However, a more thorough experimental analysis of the the top-ranked proteins potentially may reveal currently unknown mechanisms by which yeast cells respond to TOR signaling. To this end, our computational studies motivate and provide data for future experimental investigations.

\section{Sensitivity and specificity of information flow scores in predicting key transcription factors}

Top-ranked proteins in information flow analysis are highly enriched in terms of differentially expressed genes under rapamycin treatment. However, TORC1 does not directly regulate expression of these genes. This observation raises the question: which transcription factors are responsible and which intermediary elements are involved in these regulations? We answer the first question here, while deferring the latter to subsequent sections.

To find the key transcription factors that modulate the observed transcriptional response, we use two separate statistical predictors, one based on the information flow scores and the other based on the set of differentially expressed genes. These predictors allow us to assess the significance of TFs with respect to their computationally computed, top-ranked and experimentally validated targets, respectively. In the first method, we call a transcription factor relevant if a significant fraction of its target genes are highly-ranked in information flow method. Conversely, in the second method we define 


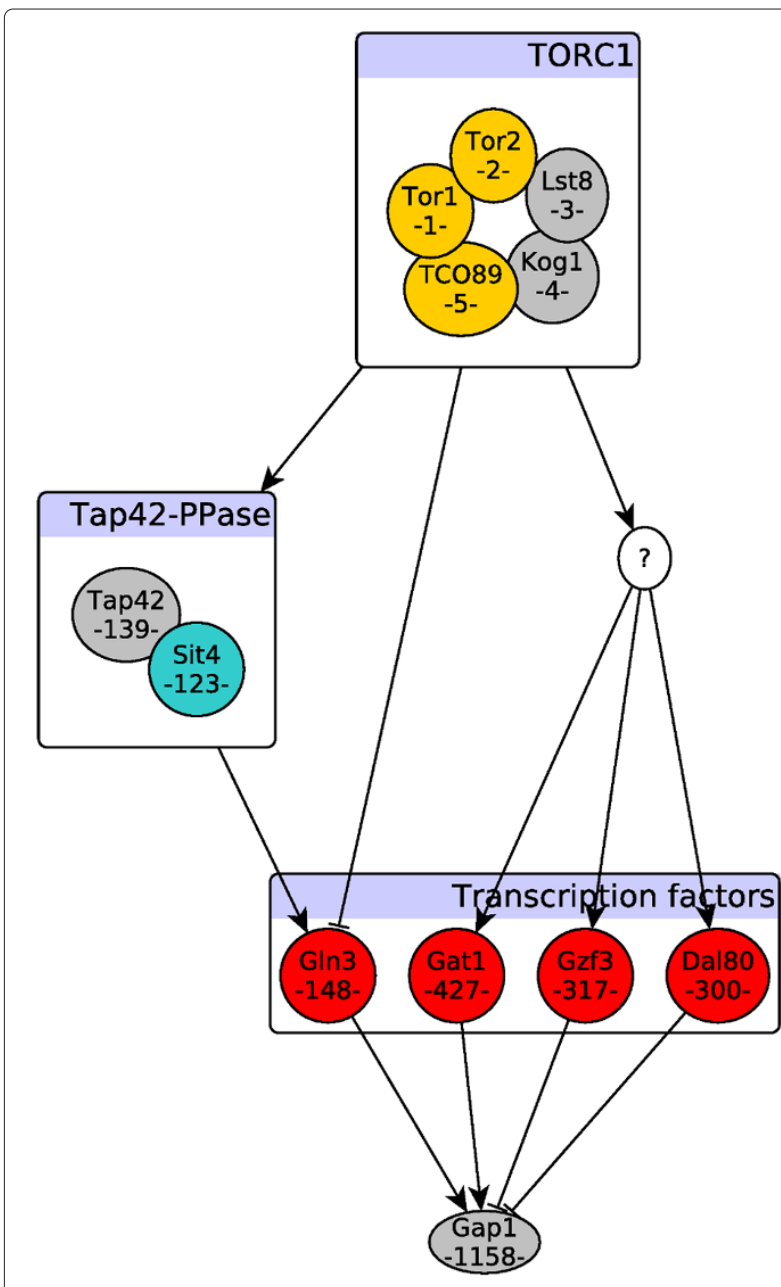

Figure 6 TORC1-dependent regulation of Gap1. The schematic diagram is based on literature evidence for the known interactions. Each node in the signaling pathway is annotated with the rank of its information flow score from TORC 1 and colored with its functional classification. Yellow nodes represent kinase associated proteins, red nodes are transcription factors, and blue node (Sit4) is a phosphatase. The rest of nodes have a default color of grey. Ranking of nodes based on their information flow scores coincides with our prior knowledge on the structure of this pathway. Top/bottom ranked nodes are discriminated using the computed cutoff value (I) based on differentially expressed genes. The "?" indicates an unknown underlying mechanism, yet to be discovered, that connects TORC1 to the rest of transcription factors.

the relevance in terms of the portion of its differentially expressed targets (please see Equations 6-8 for details).

We use $p$-value $\left(X=k_{T}\right)$ and $p$-value $\left(Y=k_{P}\right)$ and apply a cutoff value of $\epsilon=0.01$ to identify significant $\mathrm{p}$ values computed for computational and experimental predictions, respectively. At this threshold, we compute the sensitivity and specificity of information flow methods as 0.2245 and 0.9846 , respectively. The observed high specificity value suggests that if targets of a given TF are not differentially expressed, with high probability, our computational model also reports it as a negative (it will not have significant number of top-ranked targets). In other words, transcription factors that are identified as significant using information flow scores are highly precise. On the other hand, the lower sensitivity score implies that even if a TF has many differentially expressed targets, our computational method may miss it. From this, we can conclude that transcription factors that have significant numbers of top-ranked targets are high-confidence candidate(s) as downstream effectors of TORC1. However, there are cases where we may miss relevant transcription factors with a significant number of differentially expressed genes by this approach. In the next section, we propose a statistical framework to integrate information flow scores and expression profiles to reliably identify the most relevant subset of transcription factors that are involved in mediating the transcriptional response to TOR inhibition, and consequently construct the effective response network of TORC1.

\section{Identifying the most relevant transcription factors}

We now seek to integrate experimental measurements from rapamycin treatment, information flow scores, and the transcription regulatory network into a unified framework to identify the most relevant transcription factors. To this end, we introduce the notion of relevance score. Let random variable $Z$ denote the number of top-ranked positive targets, and $k_{T P}$ denote the number of top-ranked positive targets of a given TF. We define the relevance score as $-\log \left(p\right.$-value $\left.\left(Z=k_{T P}\right)\right)$. The relevance score assesses both positivity and rank of the targets for a given TF (please see Equation 10 for details). Using this approach, we identify 17 TFs with high relevance scores, which are hypothesized to be responsible for the transcriptional changes in a TORC1-dependent manner. The complete list of computed statistics for all transcription factors is summarized in Additional file 4.

The top five transcription factors are listed in Table 1. Among these top-ranked, high confidence, transcription factors, Sfp1, Gln3, and Gcn4 are well-documented downstream effectors of TORC1 [48,59-61] (please see Zaman et al. [34], Smets et al. [40], and Loewith and Hall [32] for a more comprehensive review). Sfp1 is a stressand nutrient-sensitive regulator of cell growth, responsible for mediating the expression of genes involved in ribosome biogenesis, such as RP genes and RiBi factors $[62,63]$. TORC1 mediates Sfp1-related genes by phosphorylating Sfp1 and regulating its nuclear localization [59]. Gln3, a GATA-family transcription factor, positively regulates the expression of nitrogen catabolite repression (NCR)-sensitive genes $[60,64]$. TORC1-dependent regulation of Gln3 is mediated by promoting its association with its cytoplasmic anchor protein Ure2 $[32,65]$. 


Table $\mathbf{1}$ Top-ranked transcription factors with high
confidence scores
\begin{tabular}{lccc}
\hline TF ORF & TF name & TF rank & TF confidence \\
\hline YLR403W & SFP1 & 22 & 43.5048 \\
\hline YER040W & GLN3 & 148 & 57.7734 \\
\hline YML007W & YAP1 & 618 & 24.3672 \\
\hline YEL009C & GCN4 & 638 & 4.822 \\
\hline YHR084W & STE12 & 825 & 2.9668 \\
\hline
\end{tabular}

Gcn4 is a nutrient-responsive transcription factor, which is activated upon amino acid starvation [66]. TORC1 regulates Gcn 4 by mediating its translation level in a eIF2 $\alpha$-dependent manner [32]. Interestingly, Steffen et al. [61] also proposed a critical role for Gcn4 in mediating life-span in yeast.

However, to the best of our knowledge, Ste12 and Yap1 have not been previously positioned downstream of TORC1. Ste12 is best known as a downstream target of mitogen-activated protein kinase (MAPK) signaling cascade and is responsible for regulating genes involved in mating or pseudohyphal/invasive growth [67]. Rutherford et al. [68] show that over-expression of the ammonium permease Mep2 induces the transcription of known targets of Ste12. A more recent study by Santos et al. [69] additionally positions TORC1 downstream of Mep2, which, taken together with the link between Mep2Ste12, suggests Ste12 as a potential downstream effector of TORC1. Yap1 is an AP-1 family transcription factor required for inducing oxidative [70,71] and carbon [72] stress responses, the latter is proposed to be independent of TORC1. Additionally, Yap1 expression has been shown to increase significantly during replicative aging [73]. It has been suggested that spermidine, a conserved longevity factor [74], mediates macroautophagy in a Yap1 and Gcn4 dependent manner [75]. Finally, there is a diverse set of age-related functions associated with Yap1, many of which are also attributed to TORC1. These observations suggest Yap1 as a potential candidate downstream effector of TORC1.

\section{Constructing the effective response network of TORC1}

To uncover the regulatory mechanisms that mediate the response to TOR inhibition, we construct the effective response network (ERN) of TORC1, which is illustrated in Figure 7 and is available for download as Additional file 5. Node attributes for this network are available for download separately as Additional file 6. This network consists of the most relevant TFs, together with their topranked positive targets, with a total of 1,288 regulatory interactions between 17 transcription factors and 181 target genes.
In order to better understand the functional roles of the predicted targets, we use FIDEA [76] to identify enriched GO terms under the biological process (BP) branch. Figure 8 illustrates the static word cloud of the enriched terms, as generated by FIDEA, the complete list of which is available for download as Additional file 7. Unlike the enrichment map of TORC1, which spans a variety of different functions, targets in the effective response network (ERN) are almost exclusively involved in ribosome biogenesis and the cellular translation process. Ribosome biogenesis is one of the most energy-consuming tasks in the cell that is directly linked to the rate of translation and is required for cell growth [77]. Calorie restriction, or alternatively inhibiting TORC1 by Rapamycin treatment, is known to coordinately regulate this process via a complex set of pathways involving transcription factors Ifhl, Sfp 1 , Fhl1, and Rap1 [77]. Interestingly, all four of these transcription factors are identified by our method among the top 6 TFs with the highest relevance scores (together with Gcn3 and Met4). The effective response network provides a refined view of how yeast cells re-wire various aspects of ribosome biogenesis in order to modulate cell growth. This network can be used to gain a detailed understanding of the regulatory mechanisms that are responsible for TOR-dependent transcriptional changes in yeast.

\section{Conclusions}

Understanding various processes associated with aging has important implications for the diagnosis, prognosis, and treatment of age-related pathologies. Current methods for constructing aging pathways rely on detailed experiments that study cellular response to carefully controlled signals. This process is expensive, timeconsuming, and typically restricted to specific aspects of cellular response. In this study, we presented a complementary, computational approach that aims to construct detailed aging pathways using the yeast interactome by initiating random walks at proteins that are key players in the aging process (the target of rapamycin or TOR, in this study). At the heart of our method is a rigorous statistical and computational framework that identifies significant effector proteins and provides information about the specific mechanisms associated with them.

We present comprehensive validation of our computational results through GO enrichment studies and manual curation to show that our method identifies most of the known proteins downstream from TOR, while identifying several new proteins for future experimental investigations. Additionally, we showed that information flow scores faithfully predict transcriptional changes in response to rapamycin-treatment, which validates accu- 


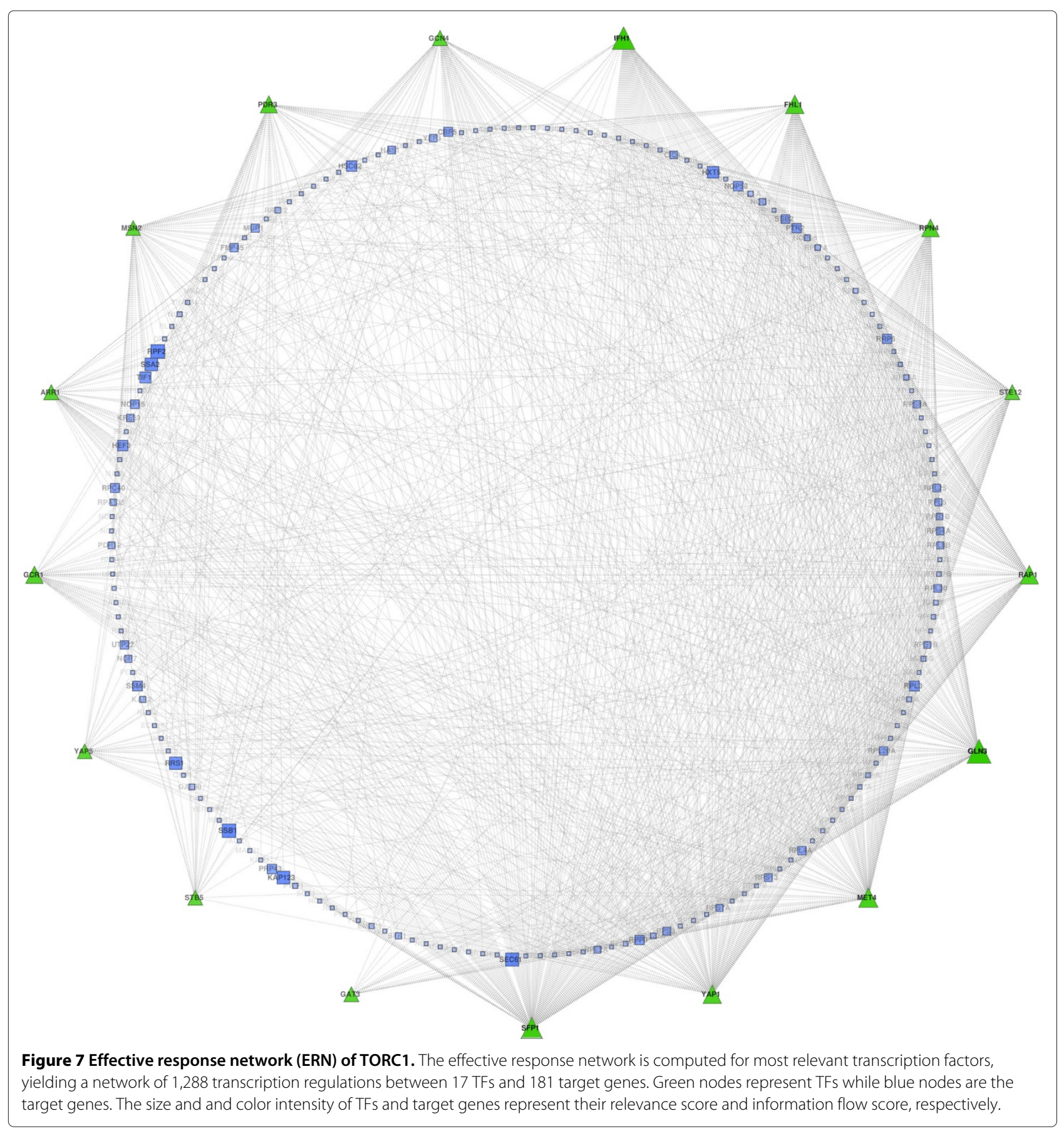

racy of predicted effectors. Furthermore, we show that the predicted targets are also enriched with proteins that are post-translationally modified (i.e., phosphorylated) in response to TOR inhibition. Finally, we constructed the effective response network of the TOR pathway. This network is hypothesized to mediate transcriptional changes in response to TOR inhibition. A direct outcome of our study is a complete dataset of proteins down- stream of TOR, their interactions, functional roles, and organization.

\section{Methods}

\section{Constructing yeast interactome}

We obtained the yeast protein-protein interactions (PPI) from the BioGRID [78] database, update 2011 [79], version 3.1 .83 , by extracting all physical interactions, except 


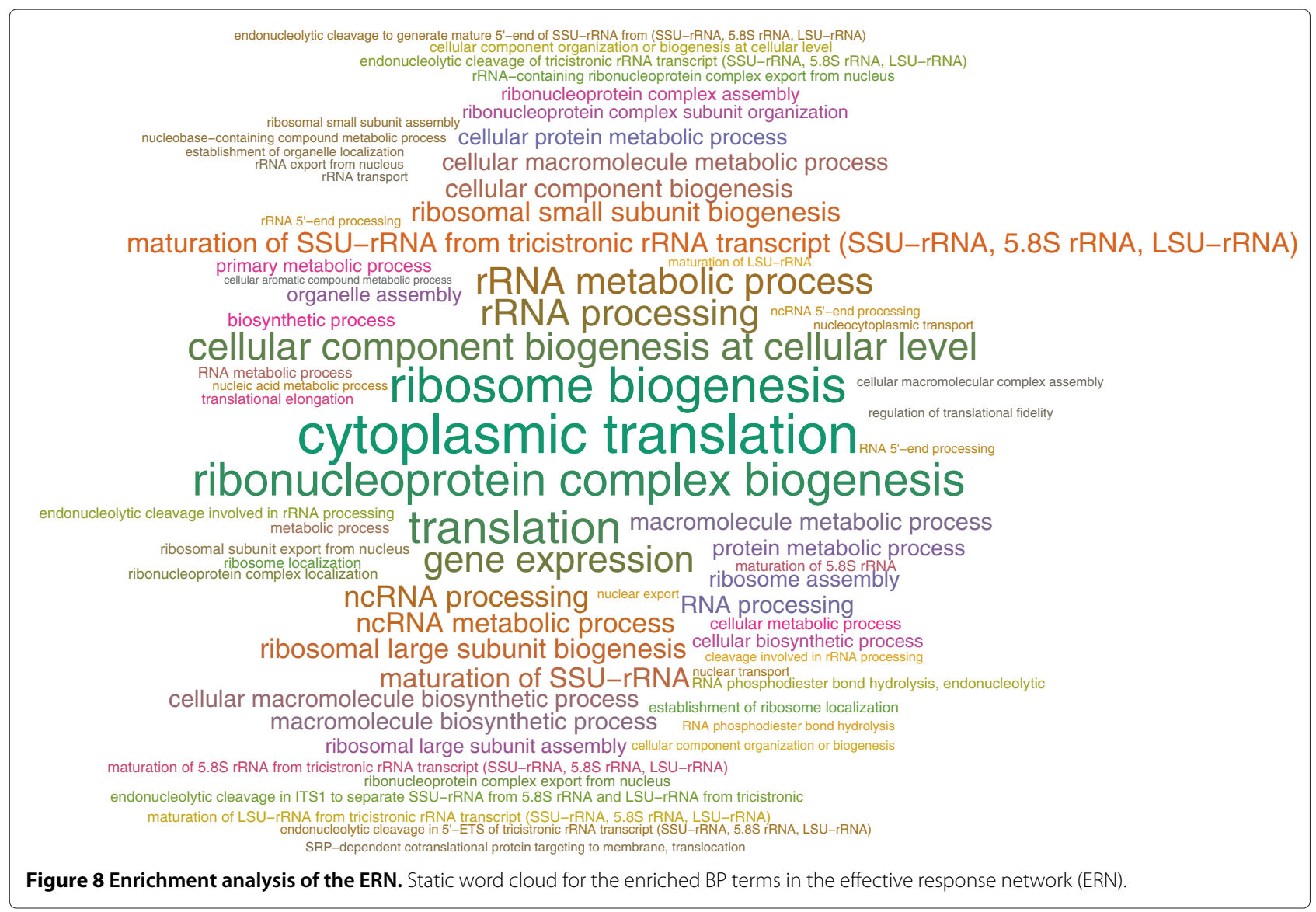

for protein-RNA interactions, and excluding interspecies and self interactions. This dataset consists of 103,619 $(63,395$ non-redundant) physical interactions among 5,691 proteins, and is available for download as Additional file 8 . We then identified the subset of interactions associated with post-translational modification (PTM), marked with the "biochemical activity" evidence code in BioGRID, resulting in 5,791 (5,443 non-redundant) biochemical activities among proteins in yeast. These are available for download as Additional file 9. Each of these interactions represents a directional enzymatic activity, where the bait protein executes the activity on the substrate hit protein. After integrating different modifications among similar pairs of proteins, we obtained 5,421 directional edges among 2,002 proteins in the yeast interactome. The bulk of these interactions (over 4,000) are the phosphorylation events identified by Ptacek et al. [80] using proteome chip technology.

We constructed the integrated network of yeast interactions, the yeast interactome, by integrating proteinprotein interactions(PPIs) and post-translational modifications(PTMs). For pairs of proteins that have both PPI and PTM, we give higher priority to PTM, since it provides a more refined description of the type of interaction.
Figure 1 illustrates an example of the integration process around the $S c h 9$ protein, which is a well-documented substrate of TORC1. The final constructed interactome is available for download as Additional file 10. This network consists of 5,287 uni-directional and 58,108 bi-directional edges (58,041 PPIs and 134 bi-directional PTMs) among 5,691 nodes. The node attributes and alternative labels for each node in the yeast interactome are also available for download as Additional file 11.

\section{Transcriptional regulatory network (TRN) of yeast}

We constructed the yeast transcriptional regulatory network (TRN) from the documented regulations in YEASTRACT [81], consisting of 48,082 interactions between 183 transcription factors (TF) and 6,403 target genes (TG). Among these 183 TFs, 179 of them have a corresponding node in the yeast interactome.

\section{Tracing information flow in the interactome}

We use a computational approach, based on a discretetime random walk process, to track directional information flow in the interactome. Similar formulations have been previously used to prioritize candidate disease genes $[82,83]$, discover network bio-markers for cancer [84], and 
identify protein complexes $[85,86]$. Additionally, there is a known correspondence between random-walk methods on undirected graphs and formulations based on circuit network models [87]. Our formulation takes into account both network distances, as well as multiplicity of paths between pairs of proteins. It also benefits from using edge directions (when available) to discriminate between upstream regulators and downstream effectors.

Let $G=(V, E)$ be a mixed graph, having both directed and undirected edges. Each node in $V$ corresponds to a protein and edge $(u, v) \in E$ iff protein $u$ interacts with protein $v$ in the integrated network. Graph $G$ can be represented using its adjacency matrix $A$, where $A_{i j}=1$, if node $i$ has a directed edge to node $j$, and is 0 otherwise. Undirected edges are replaced by a pair of directed edges in each direction. A random walk on $G$, initiated from vertex $v$, is defined as a sequence of transitions among vertices, starting from $v$. At each step, the random walker randomly chooses the next vertex from among the neighbors of the current node. The sequence of visited vertices generated by this random process is a Markov chain, since the choice of next vertex depends only on the current node. We can represent the transition matrix of this Markov process as a column-stochastic matrix, $P$, where $p_{i j}=\operatorname{Pr}\left(S_{t+1}=\right.$ $\left.v_{i} \mid S_{t}=v_{j}\right)$, and random variable $S_{t}$ represents the state of the random walk at the time step $t$.

Random walk with restart (RWR) is a modified Markov chain in which, at each step, a random walker has the choice of either continuing along its path, with probability $\alpha$, or jump (teleport) back to the initial vertex, with probability $1-\alpha$. Given the transition matrix of the original random walk process, $P$, the transition matrix of the modified chain, $M$, can be computed as $M=\alpha P+(1-\alpha) \boldsymbol{e}_{v} \mathbf{1}^{T}$, where $\boldsymbol{e}_{v}$ is a stochastic vector of size $n$ having zeros everywhere, except at index $v$, and $\mathbf{1}$ is a vector of all ones. The stationary distribution of the modified chain, $\pi_{v}(\alpha)$, defines the portion of time spent on each node in an infinite random walk with restart initiated at node $v$, with parameter $\alpha$. This stationary distribution can be computed as follows:

$$
\begin{aligned}
\boldsymbol{\pi}_{v}(\alpha) & =M \boldsymbol{\pi}_{v}(\alpha) \\
& =\left(\alpha P+(1-\alpha) \boldsymbol{e}_{v} \mathbf{1}^{T}\right) \boldsymbol{\pi}_{v}(\alpha)
\end{aligned}
$$

Enforcing a unit norm on the dominant eigenvector to ensure its stochastic property, $\left\|\boldsymbol{\pi}_{v}(\alpha)\right\|_{1}=\mathbf{1}^{T} \boldsymbol{\pi}_{v}=1$, we will have the following iterative form:

$$
\boldsymbol{\pi}_{v}(\alpha)=\alpha P \boldsymbol{\pi}_{v}(\alpha)+(1-\alpha) \boldsymbol{e}_{v}
$$

which is a special case of the personalized PageRank [88-91], with preference vector $\boldsymbol{e}_{\nu}$. Alternatively, we can compute $\pi_{v}$ directly by solving the following linear system:

$$
\boldsymbol{\pi}_{v}(\alpha)=\underbrace{(1-\alpha)(I-\alpha P)^{-1}}_{Q} \boldsymbol{e}_{v},
$$

where the right-multiplication with $\boldsymbol{e}_{v}$ simply selects column $v$ of the matrix $Q$. The factor $1-\alpha$ can be viewed as the decay factor of the signal; the higher the parameter $\alpha$, the further the signal can propagate. Let us denote by random variable $R$ the number of hops taken by random walker before it jumps back to source node $v$. Then, $R$ follows a geometric distribution with probability of success $(1-\alpha)$ and the expected (mean) length of paths taken by random walker can be computed as $E(R)=\frac{\alpha}{1-\alpha}$. In other words, if we let $\alpha=\frac{d}{1+d}$, for a given value of $d$, we expect the average length of paths taken by such a random walk to be equivalent to $d$, thus we call $d$ the depth of the random walk.

\section{Cross-validating information flow scores with the set of differentially expressed genes in response to TOR inhibition}

Given the list of gene products ranked by their information flow scores, we want to assess the enrichment of differentially expressed genes, in response to rapamycin treatment, among top-ranked proteins.

The classical approach to this problem is to select a predefined cutoff on ranks, denoted by $l$, which separates the top-ranked genes (target set) from the rest (background set), and then compute the enrichment p-value using the hypergeometric distribution. Let us denote the total number of gene products by $N$ and the total number of differentially expressed genes (true positives) by $A$. Using a similar notation as Eden et al. [57], we encode these annotations using a binary vector, $\lambda=\lambda_{1}, \lambda_{2}, \ldots \lambda_{N} \in$ $\{0,1\}^{N}$, having exactly $A$ ones and $N-A$ zeros. Let the random variable $T$ denote the number of positive genes in the target set, if we distribute genes randomly. In this formulation, the hypergeometric p-value is defined as:

$$
\begin{aligned}
p \text {-value }\left(T=b_{l}(\lambda)\right) & =\operatorname{Prob}\left(b_{l}(\lambda) \leq T\right) \\
& =H G T\left(b_{l}(\lambda) \mid N, A, l\right) \\
& =\sum_{t=b_{l}(\lambda)}^{\min (A, l)} \frac{C(A, t) C(N-A, l-t)}{C(N, l)},
\end{aligned}
$$

where $H G T$ is the tail of hypergeometric distribution, and $b_{l}(\lambda)=\sum_{i=1}^{l} \lambda_{i}$, is the number of observed positives in the target set. The drawback of this approach is that we need a predefined cutoff value, $l$. To remedy this, Eden et al. [57] propose a two-step method for computing the exact enrichment p-value, called $m H G$ p-value, without 
the need for a predefined cutoff value of $l$. In the first step of this process, we identify an optimal cut, over all possible cuts, which minimizes the hypergeometric score. The value computed in this manner is called the minimum hypergeometric ( $m H G$ ) score, and is defined as:

$$
m H G(\lambda)=\min _{1 \leq l \leq N} H G T\left(b_{l}(\lambda) \mid N, A, l\right)
$$

Next, we use a dynamic programming (DP) method to compute the exact p-value of the observed mHG score, in the state space of all possible $\lambda$ vectors with size $N$ having exactly $A$ ones (please refer to Eden et al. [57] for algorithmic details, and Eden [92] for an efficient implementation). We adopt this strategy to cross-validate our results with the transcriptome profile of yeast cells in response to rapamycin treatment. We subsequently define the enrichment plot, which illustrates the absolute value of the logarithm of the HG score as a function of cutoff percentage. The minimum hypergeometric ( $\mathrm{mHG}$ ) score can be viewed as the peak of this plot, and the corresponding exact p-value can be computed for this peak using the aforementioned DP algorithm.

\section{Assessing the sensitivity and the specificity of information flow scores}

Given an optimal cutoff length $l$ (computed for differentially expressed genes in response to TOR inhibition), which partitions nodes into top/bottom ranked proteins, together with a transcription factor (TF) of interest, $p_{i}$, we are interested in assessing the importance of $p_{i}$ in mediating the observed transcriptional response. In other words, given that $p_{i}$ has a significant number of topranked targets, how confident are we that it will also have a significant number of differentially expressed targets? Conversely, if $p_{i}$ has many differentially expressed targets, how likely is it to see its targets among top-ranked genes?

Let us denote the total number of targets of TF $p_{i}$ by $k$, and the number of its positive (differentially expressed) and top-ranked (in information flow) targets by $k_{P}$ and $k_{T}$, respectively. Let the random variable $X$ be the number of top-ranked targets, if we were uniformly distributing $k$ targets of $p_{i}$ among all genes in the yeast interactome. Similarly, let $Y$ be the number of positive targets of $p_{i}$, if we distribute positive targets uniformly. Then, we can compute the following $\mathrm{p}$-values for top-ranked and positive targets, respectively:

$$
\begin{aligned}
p \text {-value }\left(X=k_{T}\right) & =\operatorname{Prob}\left(k_{T} \leq X\right) \\
& =H G T\left(k_{T} \mid N, l, k\right) \\
& =\sum_{x=k_{T}}^{\min (l, k)} \frac{C(l, x) C(N-l, k-x)}{C(N, k)}
\end{aligned}
$$

$$
\begin{aligned}
p \text {-value }\left(Y=k_{P}\right) & =\operatorname{Prob}\left(k_{P} \leq Y\right) \\
& =H G T\left(k_{P} \mid N, A, k\right) \\
& =\sum_{y=k_{P}}^{\min (A, k)} \frac{C(A, y) C(N-A, k-y)}{C(N, k)}
\end{aligned}
$$

After correcting for multiple hypothesis testing using Bonferroni method, we use a given threshold value of $\epsilon$ and define the sensitivity and specificity for the entire set of transcription factors as:

$$
\begin{aligned}
\text { sensitivity } & =\operatorname{Prob}\left(p \text {-value }\left(X=k_{T}\right)\right. \\
& \left.\leq \epsilon \mid p \text {-value }\left(Y=k_{P}\right) \leq \epsilon\right) \\
\text { specificity } & =\operatorname{Prob}\left(\epsilon<p \text {-value }\left(X=k_{T}\right) \mid \epsilon\right. \\
& \left.<p \text {-value }\left(Y=k_{P}\right)\right)
\end{aligned}
$$

The motivation behind our approach is that the set of transcription factors with a significant number of differentially expressed targets provides us with an experimentally validated set of key factors, whereas transcription factors that have a significant number of top-ranked targets act as computational predictions for identifying the most relevant TFs. Let $T P$ be the number of identified true positives, $P$ be the total number of positives, and $F N$ be the number of false negatives. The sensitivity of a method, defined as $\frac{T P}{P}=\frac{T P}{T P+F N}$, measures the fraction of positive instances (transcription factors having a significant number of differentially expressed targets) that are also predicted using the information flow method (computational predictions). Conversely, let $T N$ be the number of true negatives identified by the method and $N$ be the total number of negatives. Specificity, formally defined as $\frac{T N}{N}=\frac{T N}{T N+F P}$, corresponds to the fraction of irrelevant TFs, computed based on the experimental dataset, that are also identified as irrelevant by our computational predictions. These two measures are closely related to type I and II errors as follows:

$$
\begin{aligned}
\text { Type I error }(\alpha) & =\text { False-positive-rate }(F P R) \\
& =1-\text { specificity } \\
\text { Type II error }(\beta) & =\text { False-negative-rate }(F N R) \\
& =1-\text { sensitivity }
\end{aligned}
$$

\section{Integrating computational predictions with experimental datasets}

Given the set of differentially expressed genes in response to rapamycin treatment, the computed information flow scores, and the transcriptional regulatory network (TRN) of yeast, we aim to construct an integrative statistical framework to identify the most relevant transcription factors with respect to mediating the transcriptional 
response to TOR inhibition, and decipher the underlying effective response network.

Let us denote the number of top-ranked positive targets of a given TF by $k_{T P}$. If we compute the probability of observing $k_{T P}$ or more positive targets among top-ranked genes, entirely by chance, we can subsequently identify the associated subset of relevant transcription factors. Let the random variable $Z$ denote the number of top-ranked positive targets, if we were randomly distributing all targets for the given TF. We can compute the p-value of $Z$ by conditioning it on the number of top-ranked targets as follows:

$$
\begin{aligned}
p \text {-value }\left(Z=k_{T P}\right)= & \operatorname{Prob}\left(k_{T P} \leq Z\right) \\
= & \sum_{x=k_{T P}}^{\min (l, k)} \operatorname{Prob}\left(k_{T P} \leq Z \mid X=x\right) \\
& \times \operatorname{Prob}(X=x) \\
= & \sum_{x=k_{T P}}^{\min (l, k)} \sum_{z=k_{T P}}^{\min \left(x, b_{l}(\lambda)\right)} \operatorname{Prob}(Z=z \mid X=x) \\
& \times \operatorname{Prob}(X=x) \\
= & \sum_{x=k_{T P}}^{\min (l, k)} H G(x \mid N, l, k) \\
& \times \sum_{z=k_{T P}}^{\min \left(x, b_{l}(\lambda)\right)} H G\left(z \mid l, b_{l}(\lambda), x\right)
\end{aligned}
$$

After correcting for multiple hypothesis testing using Bonferroni method, we define the relevance score of each TF as $-\log _{10}\left(p\right.$-value $\left.\left(Z=k_{T P}\right)\right)$, and construct the effective response network of TORC1 using the most relevant TFs, together with their top-ranked positive targets, correspondingly.

\section{Availability of supporting data}

The data sets supporting the results of this article are included within the article (and its additional files). They are also available for download from http:// compbio.soihub.org/projects/torc1. All necessary codes and datasets to reproduce the results in this paper are bundled as Additional file 12 .

\section{Additional files}

Additional file 1: Information flow scores. Excel table file (*.xls) illustrating the computed information flow scores for different proteins, sorted based on their proximity to TORC1.

Additional file 2: GO Enrichment of information flow scores. Excel table files (*.zip) illustrating the $\mathrm{GO}$ enrichment analysis using $\mathrm{mHG}$ under $\mathrm{BP}, \mathrm{CC}$, and MF branches, respectively.
Additional file 3: Enrichment map of the significant GO terms. Cytoscape file ${ }^{*}$.zip) containing the final enrichment map. Each node represents a GO term and the corresponding geneset and enrichment $\mathrm{p}$-value are encoded in its attributes. Edges are computed based on the geneset overlap among GO terms (please see www.cytoscape.org for more information about loading the file).

Additional file 4: Statistical analysis of the most relevant transcription factors (TF). Excel table file ( ${ }^{*}$.xls) illustrating the computed statistics for different transcription factors, sorted based on their proximity to TORC1.

Additional file 5: Effective response network (ERN) of TORC1. Tab separated file $\left({ }^{*}\right.$.txt) representing the edge list of the effective response network of TORC1 (corresponding nodes are marked by their systematic name)

Additional file 6: Node attributes for the effective response network. Tab-separated table file (*.txt) file containing additional attributes for the nodes in the ERN.

Additional file 7: Functional enrichment of the targets in the effective response network. Tab-separated table file ( ${ }^{*}$.txt) file containing the enriched BP terms in the ERN, identified by FIDEA.

Additional file 8: Yeast protein-protein interactions. Tab-separated table file $(*$.txt) containing the non-redundant PPI edge list (corresponding nodes are represented using their Entrez Gene ID).

Additional file 9: Yeast biochemical activities. Tab-separated table file $\left({ }^{*}\right.$. txt) containing the list of non-redundant PTM interactions (corresponding nodes are represented using their Entrez Gene ID).

Additional file 10: Yeast interactome. Simple interaction file (*.txt) containing the integrated network of the yeast interactome

(corresponding nodes are represented using their Entrez Gene ID).

Protein-protein interactions (marked with $p p$ ) are undirected while the rest of edges are directed.

Additional file 11: Node attributes for the yeast interactome. Tab-separated table file $\left({ }^{*}\right.$.txt) file containing various ID(s) for each node in the yeast interactome.

Additional file 12: Code/dataset bundle. Compressed ZIP file (*.zip) containing all codes and datasets used in this experiment.

\section{Abbreviations}

TOR: Target of rapamycin; PPI: Protein-Protein Interaction; GO: Gene Ontology.

\section{Competing interests}

The authors declare that they have no competing interests.

\section{Authors' contributions}

SM conceived the study, designed and implemented methods, performed the experiments, and prepared the manuscript. SS helped with the experimental design, as well as analyzing and interpreting the biological implications of the results. AG provided guidance relative to the theoretical and practical aspects of the algorithms, and design of proper statistical model(s) to validate the results. All authors participated in designing the structure and organization of final manuscript. All authors read and approved the final manuscript.

\section{Acknowledgements}

This work is supported by the Center for Science of Information (CSol), an NSF Science and Technology Center, under grant agreement CCF-0939370, and by NSF grants DBI 0835677 and 0800568.

\section{Author details}

${ }^{1}$ Department of Computer Science, Purdue University, West Lafayette, Indiana, USA. ${ }^{2}$ Department of Bioengineering, University of California at San Diego, La Jolla, California, USA.

Received: 23 April 2013 Accepted: 28 August 2013

Published: 30 August 2013 


\section{References}

1. Fontana $L$, Partridge $L$, Longo VD: Extending healthy life span-from yeast to humans. Science (New York, N.Y.) 2010, 328(5976):321-326.

2. Bishop NA, Guarente L: Genetic links between diet and lifespan: shared mechanisms from yeast to humans. Nat Rev Genet 2007, 8(11):835-844.

3. Kaeberlein M: Lessons on longevity from budding yeast. Nature 2010 , 464(7288):513-519.

4. Campisi J: Aging, cellular senescence, and cancer. Annu Rev Physiol 2013, 75:685-705.

5. Provinciali M, Cardelli M, Marchegiani F, Pierpaoli E: Impact of cellular senescence in aging and cancer. Curr Pharm Des 2013, 19(9):1699-709.

6. Colman RJ, Anderson RM, Johnson SC, Kastman EK, Kosmatka KJ, Beasley TM, Allison DB, Cruzen C, Simmons HA, Kemnitz JW, Weindruch R: Caloric restriction delays disease onset and mortality in rhesus monkeys. Science (New York, N.Y.) 2009, 325(5937):201-204.

7. Finkel T, Serrano M, Blasco MA: The common biology of cancer and ageing. Nature 2007, 448(7155):767-774.

8. North BJ, Sinclair DA: The intersection between aging and cardiovascular disease. Circ Res 2012, 110(8):1097-108.

9. Leritz EC, McGlinchey RE, Kellison I, Rudolph JL, Milberg WP: Cardiovascular disease risk factors and cognition in the elderly. Curr Cardiovasc Risk Rep 2011, 5(5):407-412.

10. Cruzen C, Colman RJ: Effects of caloric restriction on cardiovascular aging in non-human primates and humans. Clin Geriatr Med 2009, 25(4):733-43, ix-x.

11. Mattson MP, Wan R: Beneficial effects of intermittent fasting and caloric restriction on the cardiovascular and cerebrovascular systems. J Nutr Biochem 2005, 16(3):129-137.

12. Collier TJ, Kanaan NM, Kordower $\mathrm{JH}$ : Ageing as a primary risk factor for Parkinson's disease: evidence from studies of non-human primates. Nat Rev Neurosci 2011, 12(6):359-366.

13. Dartigues JF, Féart C: Risk factors for Alzheimer disease: aging beyond age? Neurology 2011, 77(3):206-207.

14. Duan W, Ross CA: Potential therapeutic targets for neurodegenerative diseases: lessons learned from calorie restriction. Curr Drug Targets 2010, 11(10):1281-1292.

15. Noorbakhsh F, Overall CM, Power C: Deciphering complex mechanisms in neurodegenerative diseases: the advent of systems biology. Trends Neurosci 2009, 32(2):88-100.

16. Maswood N, Young J, Tilmont E, Zhang Z, Gash DM, Gerhardt GA, Grondin R, Roth GS, Mattison J, Lane MA, Carson RE, Cohen RM, Mouton PR, Quigley C, Mattson MP, Ingram DK: Caloric restriction increases neurotrophic factor levels and attenuates neurochemical and behavioral deficits in a primate model of Parkinson's disease. ProC Natl Acad Sci USA 2004, 101(52):18171-18176.

17. Wu P, Shen Q, Dong S, Xu Z, Tsien JZ, Hu Y: Calorie restriction ameliorates neurodegenerative phenotypes in forebrain-specific presenilin-1 and presenilin-2 double knockout mice. Neurobiol Aging 2008, 29(10):1502-1511.

18. Campisi J, Andersen JK, Kapahi P, Melov S: Cellular senescence: a link between cancer and age-related degenerative disease? Semin Cancer Biol 2011, 21(6):354-359.

19. Kaeberlein M, Burtner CR, Kennedy BK: Recent developments in yeast aging. PLoS Genet 2007, 3(5):e84

20. Lin SJ, Defossez PA, Guarente L: Requirement of NAD and SIR2 for life-span extension by calorie restriction in Saccharomyces cerevisiae. Science (New York, N.Y.) 2000, 289(5487):2126-2128.

21. Kaeberlein M, Kirkland KT, Fields S, Kennedy BK: Sir2-independent life span extension by calorie restriction in yeast. PLOS Bio/ 2004, 2(9):E296.

22. Smith DL, McClure JM, Matecic M, Smith JS: Calorie restriction extends the chronological lifespan of Saccharomyces cerevisiae independently of the Sirtuins. Aging cell 2007, 6(5):649-662.

23. Kaeberlein M, Powers RW, Steffen KK, Westman EA, Hu D, Dang N, Kerr EO, Kirkland KT, Fields S, Kennedy BK: Regulation of yeast replicative life span by TOR and Sch9 in response to nutrients. Science (New York, N.Y.) 2005, 310(5751):1193-1196.

24. Heitman J, Movva NR, Hall MN: Targets for cell cycle arrest by the immunosuppressant rapamycin in yeast. Science (New York, N.Y.) 1991, 253(5022):905-909.
25. Loewith $\mathrm{R}$, Jacinto $\mathrm{E}$, Wullschleger S, Lorberg $\mathrm{A}$, Crespo JL, Bonenfant $\mathrm{D}$, Oppliger W, Jenoe P, Hall MN: Two TOR complexes, only one of which is rapamycin sensitive, have distinct roles in cell growth control. $\mathrm{Mol}$ Cell 2002, 10(3):457-468

26. Wedaman KP, Reinke A, Anderson S, Yates J, McCaffery JM, Powers $\mathrm{T}$ : Tor kinases are in distinct membrane-associated protein complexes in Saccharomyces cerevisiae. Mol Biol Cell 2003 14(3):1204-1220.

27. Reinke A, Anderson S, McCaffery JM, Yates J, Aronova S, Chu S, Fairclough S, Iverson C, Wedaman KP, Powers T: TOR complex 1 includes a novel component, Tco89p (YPL180w), and cooperates with Ssd1p to maintain cellular integrity in Saccharomyces cerevisiae. $J \mathrm{Bio} / \mathrm{Chem}$ 2004, 279(15):14752-14762.

28. Wullschleger S, Loewith $\mathrm{R}$, Hall MN: TOR signaling in growth and metabolism. Cell 2006, 124(3):471-484.

29. Kapahi P, Chen D, Rogers AN, Katewa SD, Li PWL, Thomas EL, Kockel L: With TOR, less is more: a key role for the conserved nutrient-sensing TOR pathway in aging. Cell Metab 2010, 11(6):453-465.

30. McCormick MA, Tsai SY, Kennedy BK: TOR and ageing: a complex pathway for a complex process. Philos Trans R Soc Lond B Biol Sci 2011, 366(1561):17-27.

31. De Virgilio C, Loewith R: Cell growth control: little eukaryotes make big contributions. Oncogene 2006, 25(48):6392-6415.

32. Loewith $R$, Hall MN: Target of rapamycin (TOR) in nutrient signaling and growth control. Genetics 2011, 189(4):1177-1201.

33. De Virgilio C, Loewith $R$ : The TOR signalling network from yeast to man. Int J Biochem Cell Biol 2006, 38(9):1476-1481.

34. Zaman S, Lippman SI, Zhao X, Broach JR: How Saccharomyces responds to nutrients. Annu Rev Genet 2008, 42:27-81.

35. Wei $Y$, Zheng XFS: Nutritional control of cell growth via TOR signaling in budding yeast. Methods Mol Biol (Clifton, N.J.) 2011, 759:307-319.

36. Le Couteur DG, McLachlan AJ, Quinn RJ, Simpson SJ, de Cabo R: Aging biology and novel targets for drug discovery. J Gerontol A Biol Sci Med Sci 2012, 67(2):168-174

37. Naylor RM, Baker DJ, van Deursen JM: Senescent cells: a nove therapeutic target for aging and age-related diseases. Clin Pharmacol Ther 2013, 93:105-116.

38. Binda M, Péli-Gulli MP, Bonfils G, Panchaud N, Urban J, Sturgill TW Loewith R, De Virgilio C: The Vam6 GEF controls TORC1 by activating the EGO complex. Mol Cell 2009, 35(5):563-573.

39. Neklesa TK, Davis RW: A genome-wide screen for regulators of TORC1 in response to amino acid starvation reveals a conserved Npr2/3 complex. PLoS Genet 2009, 5(6):e1000515

40. Smets B, Ghillebert R, De Snijder P, Binda M, Swinnen E, De Virgilio C, Winderickx J: Life in the midst of scarcity: adaptations to nutrient availability in Saccharomyces cerevisiae. Curr Genet 2010, 56:1-32.

41. Kuranda K, Leberre V, Sokol S, Palamarczyk G, François J: Investigating the caffeine effects in the yeast Saccharomyces cerevisiae brings new insights into the connection between TOR, PKC and Ras/cAMP signalling pathways. Mol Microbio/ 2006, 61(5):1147-1166.

42. Wanke V, Cameroni E, Uotila A, Piccolis M, Urban J, Loewith R, De Virgilio C: Caffeine extends yeast lifespan by targeting TORC1. Mol Microbiol 2008, 69:277-285

43. Chang YY, Juhász G, Goraksha-Hicks P, Arsham AM, Mallin DR, Muller LK Neufeld TP: Nutrient-dependent regulation of autophagy through the target of rapamycin pathway. Biochem Soc Trans 2009, 37(Pt 1):232-236.

44. Gasch AP, Werner-Washburne M: The genomics of yeast responses to environmental stress and starvation. Funct Integr Genomics 2002, 2(4-5):181-192.

45. Jorgensen P, Rupes I, Sharom JR, Schneper L, Broach JR, Tyers M: A dynamic transcriptional network communicates growth potential to ribosome synthesis and critical cell size. Genes Dev 2004, 18(20):2491-2505.

46. Barbet NC, Schneider U, Helliwell SB, Stansfield I, Tuite MF, Hall MN: TOR controls translation initiation and early $\mathrm{G} 1$ progression in yeast. $\mathrm{Mol}$ Biol Cell 1996, 7:25-42

47. Schmidt A, Beck T, Koller A, Kunz J, Hall MN: The TOR nutrient signalling pathway phosphorylates NPR1 and inhibits turnover of the tryptophan permease. EMBO J 1998, 17(23):6924-6931. 
48. Beck T, Hall MN: The TOR signalling pathway controls nuclear localization of nutrient-regulated transcription factors. Nature 1999 402(6762):689-692.

49. Ashburner M, Ball CA, Blake JA, Botstein D, Butler H, Cherry JM, Davis AP Dolinski K, Dwight SS, Eppig JT, Harris MA, Hill DP, Issel-Tarver L, Kasarskis A, Lewis S, Matese JC, Richardson JE, Ringwald M, Rubin GM, Sherlock G: Gene ontology: tool for the unification of biology. The Gene Ontology Consortium. Nat Genet 2000, 25:25-29.

50. Eden E, Navon R, Steinfeld I, Lipson D, Yakhini Z: GOrilla: a tool for discovery and visualization of enriched $\mathrm{GO}$ terms in ranked gene lists. BMC Bioinformatics 2009, 10:48

51. Cherry JM, Hong EL, Amundsen C, Balakrishnan R, Binkley G, Chan ET, Christie KR, Costanzo MC, Dwight SS, Engel SR, Fisk DG, Hirschman JE, Hitz BC, Karra K, Krieger CJ, Miyasato SR, Nash RS, Park J, Skrzypek MS, Simison M, Weng S, Wong ED: Saccharomyces Genome Database: the genomics resource of budding yeast. Nucleic Acids Res 2012 , 40(Database issue):D700-D705.

52. Merico D, Isserlin R, Stueker O, Emili A, Bader GD: Enrichment map: a network-based method for gene-set enrichment visualization and interpretation. PloS one 2010, 5(11):e13984.

53. Smoot ME, Ono K, Ruscheinski J, Wang PL, Ideker T: Cytoscape 2.8: new features for data integration and network visualization. Bioinformatics (Oxford, England) 2011, 27(3):431-432.

54. Hampsey M: Molecular genetics of the RNA polymerase II general transcriptional machinery. Microbio/ Mol Biol Rev 1998, 62(2):465-503.

55. Maston GA, Evans SK, Green MR: Transcriptional regulatory elements in the human genome. Annu Rev Genomics Hum Genet 2006, 7:29-59.

56. Fournier ML, Paulson A, Pavelka N, Mosley AL, Gaudenz K, Bradford WD, Glynn E, Li H, Sardiu ME, Fleharty B, Seidel C, Florens L, Washburn MP: Delayed correlation of mRNA and protein expression in rapamycin-treated cells and a role for Ggc1 in cellular sensitivity to rapamycin. Mol Cell Proteomics 2010, 9(2):271-284

57. Eden E, Lipson D, Yogev S, Yakhini Z: Discovering motifs in ranked lists of DNA sequences. PLoS Comput Biol 2007, 3(3):e39.

58. Huber A, Bodenmiller B, Uotila A, Stahl M, Wanka S, Gerrits B, Aebersold R, Loewith $R$ : Characterization of the rapamycin-sensitive phosphoproteome reveals that Sch9 is a central coordinator of protein synthesis. Genes Dev 2009, 23(16):1929-1943.

59. Lempiäinen $H$, Uotila A, Urban J, Dohnal I, Ammerer G, Loewith $R$, Shore D: Sfp1 interaction with TORC1 and Mrs6 reveals feedback regulation on TOR signaling. Mol cell 2009, 33(6):704-716.

60. Bertram PG, Choi JH, Carvalho J, Ai W, Zeng C, Chan TF, Zheng XF: Tripartite regulation of Gln3p by TOR, Ure2p, and phosphatases. J Biol Chem 2000, 275(46):35727-35733.

61. Steffen KK, MacKay VL, Kerr EO, Tsuchiya M, Hu D, Fox LA, Dang N, Johnston ED, Oakes JA, Tchao BN, Pak DN, Fields S, Kennedy BK, Kaeberlein M: Yeast life span extension by depletion of $60 \mathrm{~s}$ ribosomal subunits is mediated by Gcn4. Cell 2008, 133(2):292-302

62. Jorgensen P, Nishikawa JL, Breitkreutz BJ, Tyers M: Systematic identification of pathways that couple cell growth and division in yeast. Science (New York, N.Y.) 2002, 297(5580):395-400.

63. Marion RM, Regev A, Segal E, Barash Y, Koller D, Friedman N, O'Shea EK Sfp1 is a stress- and nutrient-sensitive regulator of ribosomal protein gene expression. Proc Natl Acad Sci U S A 2004 101(40):14315-14322

64. Courchesne WE, Magasanik B: Regulation of nitrogen assimilation in Saccharomyces cerevisiae: roles of the URE2 and GLN3 genes. J Bacteriol 1988, 170(2):708-713.

65. Urban J, Soulard A, Huber A, Lippman S, Mukhopadhyay D, Deloche O, Wanke V, Anrather D, Ammerer G, Riezman H, Broach JR, De Virgilio C, Hall MN, Loewith R: Sch9 is a major target of TORC1 in Saccharomyces cerevisiae. Mol cell 2007, 26(5):663-674.

66. Hinnebusch AG: Translational regulation of GCN4 and the general amino acid control of yeast. Annu Rev Microbiol 2005, 59:407-450.

67. Madhani HD, Fink GR: Combinatorial control required for the specificity of yeast MAPK signaling. Science (New York, N.Y.) 1997, 275(5304):1314-1317.

68. Rutherford JC, Chua G, Hughes T, Cardenas ME, Heitman J: A Mep2-dependent transcriptional profile links permease function to gene expression during pseudohyphal growth in Saccharomyces cerevisiae. Mol Biol Cell 2008, 19(7):3028-3039.
69. Santos J, MJa Sousa, Leão C: Ammonium is toxic for aging yeast cells, inducing death and shortening of the chronological lifespan. PloS one 2012, 7(5):e37090.

70. Stephen DW, Rivers SL, Jamieson DJ: The role of the YAP1 and YAP2 genes in the regulation of the adaptive oxidative stress responses of Saccharomyces cerevisiae. Mol Microbio/ 1995, 16(3):415-423.

71. Temple MD, Perrone GG, Dawes IW: Complex cellular responses to reactive oxygen species. Trends Cell Bio/ 2005, 15(6):319-326.

72. Wiatrowski HA, Carlson M: Yap1 accumulates in the nucleus in response to carbon stress in Saccharomyces cerevisiae. Eukaryot Cell 2003, 2:19-26

73. Yiu G, McCord A, Wise A, Jindal R, Hardee J, Kuo A, Shimogawa MY, Cahoon L, Wu M, Kloke J, Hardin J, Mays Hoopes LL: Pathways change in expression during replicative aging in Saccharomyces cerevisiae. J Gerontol A Biol Sci Med Sci 2008, 63:21-34.

74. Eisenberg T, Knauer H, Schauer A, Büttner S, Ruckenstuhl C, Carmona-Gutierrez D, Ring J, Schroeder S, Magnes C, Antonacci L, Fussi H, Deszcz L, Hartl R, Schraml E, Criollo A, Megalou E, Weiskopf D, Laun P, Heeren G, Breitenbach M, Grubeck-Loebenstein B, Herker E, Fahrenkrog B, Fröhlich KU, Sinner F, Tavernarakis N, Minois N, Kroemer G, Madeo F: Induction of autophagy by spermidine promotes longevity. Nat Cell Biol 2009, 11(11):1305-1314.

75. Teixeira MC, Cabrito TR, Hanif ZM, Vargas RC, Tenreiro S, Sá-Correia I: Yeast response and tolerance to polyamine toxicity involving the drug : $\mathrm{H}+$ antiporter Qdr3 and the transcription factors Yap1 and Gcn4. Microbiology 2011, 157(Pt 4):945-956.

76. D'Andrea D, Grassi L, Mazzapioda M, Tramontano A: FIDEA: a server for the functional interpretation of differential expression analysis; 2013. http://www.nar.oxfordjournals.org/cgi/doi/10.1093/nar/gkt516

77. Lempiäinen H, Shore D: Growth control and ribosome biogenesis. Curr Opin Cell Biol 2009, 21(6):855-863. http://www.ncbi.nlm.nih.gov/ pubmed/19796927

78. Stark C, Breitkreutz BJ, Reguly T, Boucher L, Breitkreutz A, Tyers M: BioGRID: a general repository for interaction datasets. Nucleic Acids Res 2006, 34(Database issue):D535-D539.

79. Stark C, Breitkreutz BJ, Chatr-Aryamontri A, Boucher L, Oughtred R, Livstone MS, Nixon J, Van Auken K, Wang X, Shi X, Reguly T, Rust JM, Winter A, Dolinski K, Tyers M: The BioGRID interaction database: 2011 update. Nucleic Acids Res 2011, 39(Database issue):D698-D704.

80. Ptacek J, Devgan G, Michaud G, Zhu H, Zhu X, Fasolo J, Guo H, Jona G, Breitkreutz A, Sopko R, McCartney RR, Schmidt MC, Rachidi N, Lee SJ, Mah AS, Meng L, Stark MJR, Stern DF, De Virgilio C, Tyers M, Andrews B, Gerstein M, Schweitzer B, Predki PF, Snyder M: Global analysis of protein phosphorylation in yeast. Nature 2005, 438(7068):679-684.

81. Abdulrehman D, Monteiro PT, Teixeira MC, Mira NP, Lourenço AB, dos Santos, S C, Cabrito TR, Francisco AP, Madeira SC, Aires RS, Oliveira AL, Sá-Correia I, Freitas AT: YEASTRACT: providing a programmatic access to curated transcriptional regulatory associations in Saccharomyces cerevisiae through a web services interface. Nucleic Acids Res 2011, 39(Database issue):D136-D140.

82. Köhler S, Bauer S, Horn D, Robinson PN: Walking the interactome for prioritization of candidate disease genes. Am J Hum Genet 2008, 82(4):949-958.

83. Navlakha S, Kingsford C: The power of protein interaction networks for associating genes with diseases. Bioinformatics 2010 26(8):1057-1063.

84. Nibbe RK, Koyutürk M, Chance MR: An integrative -omics approach to identify functional sub-networks in human colorectal cancer. PLOS Comput Biol 2010, 6:e1000639.

85. Macropol K, Can T, Singh AK: RRW: repeated random walks on genome-scale protein networks for local cluster discovery. $B M C$ Bioinformatics 2009, 10:283.

86. Maruyama O, Chihara A: NWE: Node-weighted expansion for protein complex prediction using random walk distances. Proteome Sci 2011 9(Suppl 1):S14

87. Doyle P, Snell L: Random walks and electric networks. Carus mathematical monographs. Washington: DC: Mathematical Association of America; 1984.

88. Brin S, Page L: The anatomy of a large-scale hypertextual Web search engine. Comput Netw ISDN Syst 1998, 30(1-7):107-117. 
89. Page $L$, Brin $S$, Motwani $R$, Winograd $T$ : The PageRank citation ranking: bringing order to the web. Technical Report 1999-66, Stanford InfoLab 1999.

90. Jeh G, Widom J: Scaling personalized web search. Technical Report 2002-12, Stanford InfoLab 2002.

91. Haveliwala TH: Proceedings of the 11th International Conference on World Wide Web, WWW'02. 2002:517-526.

92. Eden E: Discovering motifs in ranked lists of DNA sequences. $P h D$ thesis. Technion - Israel Institute of Technology 2007.

doi:10.1186/1752-0509-7-84

Cite this article as: Mohammadi et al:: Inferring the effective TOR-dependent network: a computational study in yeast. BMC Systems Biology 2013 7:84.

Submit your next manuscript to BioMed Central and take full advantage of:

- Convenient online submission

- Thorough peer review

- No space constraints or color figure charges

- Immediate publication on acceptance

- Inclusion in PubMed, CAS, Scopus and Google Scholar

- Research which is freely available for redistribution

Submit your manuscript at www.biomedcentral.com/submit
C Biomed Central 